Meta

Journal des traducteurs

Translators' Journal

\title{
La traduction dans l'entreprise et l'entreprise de traduction à Toronto
}

\section{François Gauthier}

Volume 21, numéro 1, mars 1976

La traduction et l'entreprise

URI : https://id.erudit.org/iderudit/002829ar

DOI : https://doi.org/10.7202/002829ar

Aller au sommaire du numéro

Éditeur(s)

Les Presses de l'Université de Montréal

ISSN

0026-0452 (imprimé)

1492-1421 (numérique)

Découvrir la revue

Citer cet article

Gauthier, F. (1976). La traduction dans l'entreprise et l'entreprise de traduction à Toronto. Meta, 21(1), 42-63. https://doi.org/10.7202/002829ar d'utilisation que vous pouvez consulter en ligne. 


\section{La traduction dans l'entreprise et l'entreprise de traduction à Toronto}

C'est dans un local aimablement prêté par les autorités du Groupe Noranda que j'ai rencontré quatre chefs de services de traduction de Toronto pour discuter des plaisirs et souffrances des traducteurs cuvrant dans cette ville que l'on qualifie de reine. Certaines obligations l'ayant empêché de participer à la table ronde, M. Gérard Gauthier, directeur du service français de la société Manu Vie, a quand même tenu à exprimer ses vues dans une longue lettre que l'espace ne nous permet pas de reproduire. Il $\mathrm{y}$ traite de certains grands thèmes dont les participants à la table ronde discuteront, en particulier de l'incompréhension des unilingues anglophones et de l'aménagement paysager.

Les participants à l'entretien, identifiés à chaque intervention par leurs initiales, étaient les suivants :

D. L. : M $^{\text {lle }}$ Danielle Lord,

Chef du service de traduction, Groupe Noranda.

J. G. D. : M. Jean-Guy Dallaire,

Chef du service de traduction, La Confédération, C Cie d'assurance-vie.

L.F.: M. Laurier Filion,

Chef du service français du catalogue Eaton.

C. M. : M. Charles Metz,

Président, Holt French Translating Bureau Limited.

F. G. : François Gauthier,

Représentant Meta.

FrançoIs GaUthiER

F. G. - Les traducteurs ont-ils une place dans l'organigramme qui leur permet de parler d'égal à égal avec leurs clients à l'intérieur de l'entreprise ?

L. F. - Notre client c'est notre employeur. Quant à la place dans l'organigramme, il faudrait faire une distinction entre la place que le service de traduction de l'entreprise devrait occuper et la place qu'il occupe réellement dans l'organigramme, sans parler du rôle que le service de traduction joue pour rehausser le prestige et la réputation dont l'entreprise jouit auprès du public francophone: 
l'entreprise étant anglophone, elle compte sur son service de traduction pour la représenter sur le marché du Canada français.

F. G. - En somme il s'agit d'un service de publicité.

L. F. - Presque ça. Et à cause de cela, le service de traduction devrait bénéficier d'une certaine autonomie et occuper une place dans l'organisme qui lui permettrait d'avoir des rapports directs avec la haute direction; pour bien remplir ses fonctions, il lui faut connaître les vues et les principes de la direction afin de les traduire correctement. Mais on sait que ce n'est pas comme cela que ça se passe. Un service de traduction dans une entreprise est ordinairement rattaché à un autre service, généralement le service de publicité. C'est le cas chez nous. Nous suivons un cours de rédaction parallèle à celui du service de rédaction anglais et nos deux services répondent directement au chef de la publicité. Si on veut en connaître davantage au sujet de la politique de la maison, c'est à lui qu'on s'adresse. Les cas sont rares où l'on doive se rendre au vice-président ou au président ou à un administrateur.

D. L. - Nous sommes rattachés aux relations publiques et nous dépendons du directeur de ce service.

J. G. D. - Jusqu'à il y a un an environ, nous dépendions des services administratifs. Ensuite il y a eu tout un remaniement dans la compagnie et jusqu'à la semaine dernière, nous dépendions du service aux assurés. Maintenant nous répondons directement au vice-président. C'est tout un changement : nous évitons tout le cheminement que nous avions à faire avant d'arriver à cette personne et les délais interminables.

D. L. - Mes clients sont les différentes compagnies du Groupe Noranda. Celui-ci comprend des usines partout au Canada. Ces clients s'adressent à moi la plupart du temps, quand ils savent que j'existe, sinon au directeur des relations publiques qui m'apporte le travail. À partir de ce moment, je peux communiquer directement avec le client et le consulter si c'est nécessaire.

F. G. - Cette façon de procéder vous convient-elle ? Est-ce que le service de traduction ne pourrait pas jouir d'une plus grande autonomie?

D. L. - Évidemment, mais comme nous ne sommes que deux, on peut se demander comment nous pourrions justifier la création d'un service de traduction autonome. Il n'y a pas de budget pour la traduction. Mon salaire, mes voyages, mes dépenses sont tirés de divers comptes des relations publiques.

F. G. - Chez vous, M. Dallaire, tout semble bien aller puisque vous êtes en relation directe avec le vice-président.

J. G. D. - On nous a trimbalés d'un service à l'autre et, chaque fois, il fallait tout recommencer à zéro, tout expliquer. La traduction, ils ne savent pas du tout ce que c'est. D'abord ils pensent parfois qu'un travail qu'ils ont mis trois semaines à préparer peut être traduit dans une demi-heure. Les changements récents sont venus à la suite d'une longue absence de notre ancien chef. J'ai alors dû faire affaire avec son supérieur immédiat qui était le vice-président. Il y a donc eu une certaine initiation à ce moment-là et maintenant je n'ai pas cette rééducation à faire. 
F. G. - La situation actuelle va-t-elle durer ?

J. G. D. - Je le crois. Et j'ai bien l'impression que ce sera l'occasion d'améliorer le statut du service de traduction. S'il survient des problèmes ou s'il y a des plaintes, au lieu d'aller voir un anglophone, on pourra s'adresser directement à moi pour obtenir les réponses. Autrefois, la personne consultée devait me voir parce qu'elle-même ne pouvait répondre. Nous avons donc plus d'autonomie.

F. G. - Est-ce que le service de traduction pourrait rendre d'autres services que celui qu'il rend actuellement, par exemple, celui de conseiller linguistique auprès de l'entreprise?

L. F. - Dans notre cas nous rendons d'autres services. Nous devons étudier, nous tenir au courant des particularités du marché canadien-français et en informer notre employeur. Dans nos publications françaises, nous devons éviter de pécher contre ce que l'employeur fait. Prenons par exemple la Loi de la protection du consommateur dans laquelle il y a certains règlements qui touchent la publicité d'une entreprise et la Loi sur l'étiquetage : il faut que le français occupe une place aussi avantageuse que l'anglais. Nous avons dû refaire un grand nombre d'étiquettes à cause de cette loi. Il faut être au courant de ces lois et règlements. Si on nous apporte quelque chose qui va à l'encontre des exigences du marché canadienfrançais, nous indiquons la bonne marche à suivre pour que la loi soit respectée. Il y a aussi la Loi 22 . Des instructions rédigées en anglais auxquelles est attaché un simple petit feuillet abrégé pour le français, ça ne va plus. Il faut que tout soit bilingue, et l'entreprise doit prendre les mesures nécessaires pour que ce soit ainsi. Il faut done la conseiller sous ce rapport : il s'agit d'un service que l'on doit rendre, me semble-t-il, puisque nous sommes plus en mesure de connattre ces choses que l'entreprise qui est anglophone.

J. G. D. - Même à l'intérieur de la compagnie : nous avions, à l'intention de nos agents, plusieurs manuels qui étaient rédigés uniquement en anglais. Depuis la Loi 22, nous avons dû entreprendre la traduction de tout cela. Il s'agit qu'une personne demande un texte français pour qu'on doive le lui donner. Notre compagnie a préféré prendre les devants au lieu d'avoir à tout faire d'un seul coup.

F. G. - Est-ce conseillée par vous que l'entreprise a décidé d'effectuer immédiatement ces traductions à cause des lois ?

J. G. D. - Quand j'ai pris connaissance de la loi, j'ai posé la question : «Est-ce qu'on va marcher ou si on ne marchera pas ? » On m'a dit : «Voyons ce qui va se produire. $\gg$ Nous avons eu des pressions de la part de nos bureaux de Montréal et de Québec, de même que des succursales. Ces cö̈ncidences ont fait bouger l'administration.

D. L. - Je pense qu'à cause de la Loi 22 on a beaucoup plus de travail.

J. G. D. - Chez nous le volume a triplé. Nous étions sept traducteurs ; maintenant nous sommes neuf à Toronto et deux à Montréal.

D. L. - Depuis l'adoption de la Loi 22 nous avons eu à traduire beaucoup de brochures à l'intention des vendeurs. Nous n'avions jamais eu de telles brochures auparavant, étant donné que les vendeurs utilisaient de la documentation anglaise. 
L. F. - Il y a un autre service que l'on pourrait rendre et que nous chez Eaton, rendons maintenant. Nous exigeons que les traductions faites en dehors de notre service nous soient soumises avant d'être publiées. Chez nous, les acheteurs disent aux manufacturiers que les instructions doivent être bilingues. Certains vont dire : «Faites faire la traduction et envoyez-nous la facture. » D'autres se chargent eux-mêmes de fournir les instructions bilingues. C'est le cas surtout chez les manufacturiers américains. Les instructions sont donc traduites par eux et mises dans l'emballage dans lequel se présente l'article en magasin. Le client de Québec ou de Mostréal qui achète l'article trouve la traduction et que dit-il ?: «Toronto French ». Simplement parce que le siège social de notre entreprise se situe à Toronto. La traduction effectuée dans les divers services de traduction des entreprises et dans des entreprises de traduction de Toronto est généralement bonne ; mais ce n'est pas l'impression qu'ont les clients lorsqu'ils voient de telles choses. En exigeant que la traduction soit faite par un service compétent, on rend un service à la compagnie. La réputation de Toronto a été ternie injustement par des choses comme ça.

J. G. D. - Chez nous, ce seraient plutôt différentes personnes dans la compagnie qui, prétendant parler français, vont essayer de faire de la correspondance en français. Si en consultant un dossier nous prenons connaissance de ce fait, nous nous plaignons au supérieur de cette personne et tentons de lui faire reconnaître que tout ce qui sort en français doit passer par nous. Il arrive aussi assez souvent que des clients du Québec ou du nord de l'Ontario s'adressent à nous pour accélérer le service. Toutes les communications françaises passent maintenant par notre service. Nous nous chargeons d'obtenir pour le client les renseignements nécessaires et de les lui transmettre. Le contact avec le client se fait en français.

D. L. - C'est la même chose chez nous pour les gens qui s'essaient à faire de la traduction; quand on s'en aperçoit, on agit en conséquence : on avertit le supérieur de ces personnes.

J. G. D. - Vous lancez un avertissement ; pendant quelques mois c'est calmé, puis, tout à coup, ça recommence : il faut revenir à la charge.

D. L. - Ils s'imaginent que dès que quelqu'un est capable de parler français il peut faire de la traduction. Souvent ils ne savent pas écrire le français. Il arrive aussi que certaines brochures traduites par Dieu-sait-qui, il y a plusieurs années, soient envoyées à la réimpression sans que je les aie vues pour vérifier leur qualité. Tout ce que je reçois, ce sont les épreuves et c'est toute une histoire pour faire changer un texte.

J. G. D. - Il arrive souvent chez nous que les brochures explicatives au sujet des assurances collectives soient traduites par nous, puis envoyées pour approbation à l'entreprise qui souscrit une assurance collective. C'est cette dernière qui paye pour la traduction et l'impression. Je donne un exemple : dans un texte, on parle de mutilation accidentelle. La compagnie remplace mutilation par démembrement. Cette correction m'est envoyée, je remets mutilation. Sur le texte imprimé, je trouve encore démembrement. On vient ensuite me dire: «Le client a raison : c'est sa brochure, son nom va apparaître dessus, c'est lui qui paye. $\gg$ Mais sur la brochure on lit quand même: La Confédération, Compagnie d'Assurances. Ces choses-là sont très embêtantes. 
F. G. - Je crois que cette anecdote peut nous servir d'entrée en matière pour les entreprises de traduction. Enfin, nous pensons à vous M. Metz? Chez vous, comment se font les relations avec les clients?

C. M. - Je veux faire un bref historique de notre entreprise. J'ai eu la chance d'entrer dans une compagnie qui existait déjà depuis longtemps. J'ai commencé ici en 1953 et je suis président de la compagnie depuis 1960: par conséquent c'était une affaire qui marchait déjà et je n'ai eu qu'à poursuivre le travail. Parmi les clients que nous avons à l'heure actuelle, il y en a que nous avions déjà il y a 22 ans. À cette époque certains d'entre eux recouraient à nos services depuis 15 ans. $\mathrm{Il}$ y a donc un noyau de clients établis et ils ont confiance dans nos traductions, en principe. Ils viennent chez nous depuis toujours parce qu'ils s'attendent à avoir une certaine qualité de travail, un certain service. Ces clients, il faut les satisfaire et c'est pourquoi on s'efforce d'avoir de bonnes relations. Mais tout dépend des compagnies. Pour certaines, le traducteur c'est simplement un petit employé à leur service.

F. G. - Une espèce de gratte-papier.

J. G. D. - Comme j'ai déjà entendu : «A pain in the neck».

C. M. - Exactement. Je pense à une grande société de produits alimentaires qui m'a fait venir un jour dans ses bureaux. Il y avait là le chef de la publicité, le vice-président à ceci et le vice-président à cela. Ils m'ont dit : "Voilà Charles, chaque fois que nous aurons un problème en français, on vous appelle, vous venez ici et on discute. » «Non, non, non, je regrette ! Impossible! Pour la bonne raison que je n'ai pas qu'un client. Supposons qu'on me fasse faire cela partout ! $\gg$ II a fallu que je leur fasse comprendre que tout le travail se fait dans nos bureaux, par le personnel du bureau ; s'il faut discuter après, soit au téléphone, soit de vive voix, si c'est quelque chose d'important, d'accord. Je ne suis pas le petit larbin à leur service. Ils ont très bien compris quel genre d'entreprise on était. Mais c'est quand même l'exception.

J. G. D. - C'est sans doute au début de vos relations avec ce client-là ?

C. M. - C'était déjà un vieux client. Mais il y avait deux francophones dans cette maison. L'un travaillait aux cuisines et l'autre était plus ou moins relié à la vente. Ces personnes faisaient de petits changements dans nos textes. Leurs supérieurs anglophones ne comprenaient pas pourquoi. Ainsi, nous avions à traduire les étiquettes qui devaient apparaître sur les emballages d'un produit qui ne comportait pas de fraises, mais qui goûtait la fraise. Nous avons donc écrit : pouding à la fraise, puisqu'il avait simplement un arôme de fraise. Lors de la réunion dont je parlais tout à l'heure, on soulève ce point : «Nous avons eu assez d'ennuis avec cette traduction; nous avons été obligés de détruire pour $\$ 10000$ de boîtes de ce produit. » Je leur demande qui a fait détruire ce stock. Un francophone de l'entreprise me répond qu'on a regardé dans six livres de cuisine et qu'on n'a trouvé à la fraise nulle part. Ils avaient pris la mauvaise décision tout simplement parce qu'ils n'étaient pas venus me consulter au moment voulu: ils avaient fait confiance aux francophones de l'entreprise. 
J. G. D. - C'est comme cela qu'on obtient le résultat de l'histoire que je racontais tantôt au sujet de démembrement. On envoie notre traduction à Montréal et c'est l'autre qui l'emporte. Surtout si c'est un directeur de succursale. Parce qu'il est au Québec, il sait ce qu'il faut dire !

C. M. - Tout cela pour vous dire que les relations avec les clients ne sont pas toujours idéales. On nous consulte, mais en tant que bureau de traduction, nous ne sommes ni plus ni moins que des pigistes à cette différence qu'au lieu d'être seuls, nous sommes une entreprise. Mais il y a tout de même une certaine satisfaction à savoir qu'on remet un travail convenable, qu'il n'y a pas trop d'ennuis, pas trop de réclamations de la part des ménagères du Québec.

F. G. - Est-ce que vous croyez que les autres entreprises de traduction de Toronto ont aussi à souffrir de cette perception de la traduction par les clients ?

C. M. - Il y a assez peu de relations entre les différents traducteurs de Toronto, et la chose est vraie aussi entre les différents bureaux de traduction. Il y a bien certains de mes concurrents à qui je parle au téléphone de temps en temps; on se dit certaines choses sur les clients, mais pas tout : nous sommes concurrents, n'est-ce pas ? D'après ce qu'on entend à droite et à gauche, je sais par exemple qu'il y a des chefs de bureau qui font très peu de traduction. Moi-même, au cours des années, j'ai essayé différents systèmes, et celui auquel je suis arrivé maintenant me fait faire plus de traduction que jamais. Nous sommes un bureau relativement petit: : seulement deux traducteurs. Il fut un temps où j'avais un associé et trois, même quatre traducteurs que j'essayais de former. Je vais peut-être paraître un peu pessimiste ou sceptique, mais je me suis rendu compte à ce moment-là qu'il est impossible de former beaucoup de traducteurs.

Il faut qu'un traducteur sache travailler dans des domaines très différents. Très souvent encore, ce qui manquait, c'était la connaissance de l'anglais, et cela s'applique non seulement aux gens qui venaient d'Europe, mais aussi aux gens du Québec. Les jeunes qui sortent de l'école ne savent pas l'anglais si bien que ça. Il y a aussi des lacunes au point de vue français et surtout, les fautes que je tenais absolument à voir corrigées ne l'étaient pas. Alors je me suis dit que ça ne valait pas la peine, que dans cette affaire nous étions deux à perdre notre temps, lui et moi.

J. G. D. - Vous deveniez correcteur d'épreuves en somme ?

C. M. - J'étais surtout réviseur à ce moment-là. Je me suis aperçu que c'était plus satisfaisant, plus agréable de faire à peu près cinquante à soixante pour cent du travail moi-même plutôt que de le faire faire par des gens qui n'étaient pas vraiment compétents.

L. F. - C'est possible de faire cela quand le bureau de traduction est relativement petit. Nous sommes quinze chez nous, ce serait impossible de procéder ainsi. Nous formons d'abord nos traducteurs : il est rare que nous puissions embaucher un traducteur qualifié capable de faire un travail fini dès le premier jour. Comme nous devons traduire dans un grand nombre de domaines, après un certain temps, nous encourageons nos gens à suivre des cours spécialisés, par exemple, des 
cours de photographie, sans parler des cours de traduction. Ces cours ont pour but de bien faire connaître la matière. On peut décrire un appareil photo de quarante ou cinquante dollars : on sait que le client se fiche des renseignements techniques. Mais s'il s'agit d'un appareil de quatre ou cinq cents dollars, ce n'est pas la même chose.

D. L. - Les employés suivent-ils ces cours en anglais ? Les cours de photographie par exemple?

L.F. - Certainement qu'ils les suivent en anglais. Ils ne se donnent pas en français à Toronto.

D. L. - Et la terminologie française ?

L. F. - La terminologie française est un travail d'équipe. Nous fabriquons les lexiques par sujets, par exemple, la photographie, les armes à feu. C'est la documentation. Mais il est aussi important que le traducteur connaisse la marchandise. S'il ne la connaît pas, même s'il a la terminologie, il va se tromper royalement.

J. G. D. - Chez nous, j'ai peut-être seulement cinq pour cent de mon temps à consacrer à la traduction et d'ordinaire, c'est seulement quand mes traducteurs viennent me consulter. Tout le reste, c'est de l'administration.

L. F. - Nous avons deux réviseurs et je travaille surtout avec eux. Je leur dis ce que l'on veut, quel niveau de qualité il faut atteindre ; je leur indique la terminologie à employer ou à ne pas employer. Lorsqu'ils révisent les textes des traducteurs, le traducteur et le réviseur doivent travailler à l'amiable.

C. M. - À propos de ce que j'ai dit tout à l'heure au sujet des traducteurs qui étaient impossibles à former, il faudrait que je donne quand même une précision : premièrement, cela indique la faiblesse de notre méthode de recrutement ici à Toronto, surtout celle qui existait il y a dix ou quinze ans. C'était pour ainsi dire impossible de trouver de bons traducteurs. Il s'agissait toujours de débutants et à priori vous ne savez pas si une personne va faire un bon traducteur ; c'est un coup de chance. Il n'y a vraiment qu'une personne sur cinq qui réussira. Je dois dire aussi que $j$ 'ai eu de très bons traducteurs, mais au bout de deux ou trois ans ils sont repartis en Europe, à Montréal ou à New York. Il y avait donc tout un travail de formation qui était perdu pour le bureau, et cela s'est produit plusieurs fois.

F. G. - Vous n'êtes maintenant que deux traducteurs; yous avez essayé d'augmenter votre personnel sans succès. Serait-il possible pour une entreprise de traduction connaissant bien ses clients de spécialiser chacun de ses traducteurs dans un certain nombre de disciplines ?

C. M. - Théoriquement, c'est possible. Dans la pratique, c'est très difficile. En plus des deux traducteurs, nous avons un correcteur d'épreuves à plein temps. Autrement dit, tout ce qui est imprimé à Toronto n'est pas corrigé chez l'imprimeur, mais au bureau de traduction. Mon collègue et moi faisons la plus grande partie de notre travail au dictaphone, il nous faut done une dactylo pour taper le travail au brouillon d'abord et au propre après révision par les traducteurs. Nous avons aussi deux secrétaires qui s'occupent de la comptabilité, de la facturation, de la réception, 
etc. Autrement dit, un personnel administratif très compétent. Je n'ai pas à corriger de fautes d'orthographe chez mes dactylos; je ne dis pas qu'il n'y en a pas, mais j'essaye qu'il y en ait le moins possible et je les forme sous ce rapport. Chaque personne est entièrement responsable du travail qu'elle effectue.

F. G. - M. Dallaire, comment votre service fonctionne-t-il ?

J. G. D. - Comme chef de service, je m'occupe surtout de l'administration et de la consultation. Comme on peut prévoir l'arrivée de certains travaux, le rapport annuel ou le journal de l'entreprise, il y a des personnes qui sont affectées uniquement à ces tâches.

F. G. - Est-ce que ces personnes appartiennent à des niveaux différents ? Par exemple, réviseur, traducteur?

J. G. D. - J'ai un superviseur qui s'occupe des traductions techniques surtout et fait la navette entre la traductrice principale et moi. Il joue le rôle de réviseur et m'aide à déterminer les dates limites pour la remise des travaux techniques; il reçoit aussi nos clients et s'entend sur les délais à observer. En mon absence, il prend la relève. Il n'y a que trois personnes qui se partagent les travaux techniques. Nous en sommes arrivés là parce que, nous aussi, nous devons former les traducteurs à partir de zéro. Les quatre que j'ai engagés cette année ne sont pas prêts encore à toucher aux travaux techniques.

F. G. - Qui forme les nouveaux employés ?

J. G. D. - C'est surtout le réviseur. Nous avions auparavant un système où chacun se spécialisait; je préfère maintenant amener chacun à toucher un peu à tout. Avec l'ancien système, quand une personne partait, on pouvait être désorganisé pendant un an. Maintenant, quelqu'un est toujours prêt à prendre la relève.

D. L. - Nous ne sommes que deux. Je révise toutes les traductions de mon adjoint. Nous discutons ensemble des corrections, nous faisons de la recherche.

F. G. - Votre service de traduction est-il suffisant pour répondre à tous les besoins?

D. L. - En ce moment oui. Mais le travail augmente tout le temps. À l'heure actuelle nous tapons chacun les textes que nous traduisons. La prochaine personne à engager sera sans doute une dactylo, ce qui nous permettrait de faire plus de traduction.

F.G. - Vous ne pouvez engager de personnel sans passer par la voie hiérarchique?

D. L. - Non. Il m'a fallu six mois de négociations avant d'obtenir l'autorisation d'engager quelqu'un.

F. G. - Et chez vous, M. Filion ?

L. F. - Notre service nous suffit actuellement ; cependant, à cause de la Loi 22 , nous avons plus de travail. Nous traduisons pour d'autres services de l'entreprise en plus de celui du catalogue. Ces autres travaux sont devenus tellement 
nombreux que j'ai dû en faire part à la direction. Nous nous sommes entendus pour que le bureau de Montréal apporte les modifications qu'il faut pour absorber ce surcroît de travail. Le personnel que nous avons en ce moment pour la traduction du catalogue est donc suffisant.

J. G. D. - Nous avons eu un peu la même expérience vers la fin de l'année dernière ; nous avons commencé à être surchargés au moment de la parution de la Loi 22, si je me souviens bien. Nous avons essayé de survivre en faisant du temps supplémentaire. J'ai donc demandé au moins un autre employé. On m'a répondu : «Attendez au milieu de janvier; nous verrons alors s'il ne s'agit pas simplement d'une surcharge de fin d'année. » Vers la mi-janvier, j'ai dû revenir à la charge, expliquer que je ne pouvais plus respecter les délais. C'est à ce moment qu'on m'a permis d'engager quelqu'un.

\section{F. G. - Est-il facile de trouver du personnel ?}

J. G. D. - Presque impossible. Je cherche un traducteur depuis un mois et demi et je n'ai personne. Il ne semble pas y avoir de juste milieu. Vous avez des gens qui veulent devenir traducteurs à 7000 dollars, vous en avez d'autres qui ont peut-être un cours de traduction ou qui sont en première année du cours de traduction ici à Toronto et à qui on a dit que le poste de traducteur était payant : ils veulent 15000 dollars! J'ai employé une traductrice pour le bureau de Montréal à 9400 dollars. Après trois mois, elle a démissionné : une banque lui avait offert un salaire supérieur. À cause de la Loi 22 , actuellement c'est la course aux traducteurs ; pour la garder, il aurait fallu que je paye la nouvelle venue plus cher que ceux qui ont déjà trois ou quatre ans d'expérience et qui connaissent le travail.

\section{F. G. - À Toronto le réservoir de traducteurs est-il suffisant ?}

L. F. - Il y a une pénurie de traducteurs compétents et qualifiés.

J. G. D. - Il y a une pénurie et je crois aussi qu'on ne paye pas suffisamment. Nous serions prêts à payer 9400 dollars pour une personne que nous irions chercher à Montréal, mais à salaire égal, celle-ci préfère rester à Montréal. La rémunération est donc trop faible au début. Il y a aussi le facteur concurrence. Tous les bureaux cherchent des traducteurs. Étant donné qu'on doit engager des débutants, il y a aussi un problème lorsque les travaux commencent à être un peu plus techniques. Un nouveau traducteur ne produira pas le même volume de travail qu'un autre en place depuis longtemps. Quand j'ai pris charge du service en 1969, tous les traducteurs avaient moins d'un an d'espérience sauf un. Il fallait que je revoie presque tout. Maintenant je ne fais plus de révision et chaque traducteur produit de quatre à cinq fois plus de travail qu'à l'époque. Et la qualité est certainement meilleure.

F. G. - Le recrutement dans les entreprises de traduction est-il un problème ?

C. M. - C'est très curieux, mais quelquefois c'est un coup de chance. Quelqu'un vient se présenter chez vous, vous avez justement besoin de quelqu'un à ce moment-là, il se trouve que le candidat est très bon. Par contre, vous mettez des annonces dans les journaux, des annonces très grandes parce que vous pensez que plus elles seront grandes plus vous obtiendrez de résultats, vous obtenez une 
cinquantaine de réponses, vous choisissez d'en voir cinq et sur les cinq... Vous avez en outre des cas de conscience : par exemple, une personne qui travaille déjà dans la traduction. Elle est relativement satisfaite là où elle travaille, cependant, si elle trouvait un poste de traducteur dans une maison comme la nôtre, à 1000 ou 500 dollars de plus, elle serait disposée à changer. Il y a un très gros risque : en supposant que le candidat soit bon traducteur là où il travaille, cela ne veut pas dire qu'il réussira dans la traduction publicitaire, la traduction technique. Je me pose alors la question suivante : ai-je raison de faire perdre sa place à ce traducteur, de l'ernbaucher, pour lui dire au bout de six mois : "Écoutez, vous ne faites pas l'affaire, je regrette? »

L. F. - En effet, un traducteur versé dans le domaine de l'automobile ne ferait pas nécessairement un bon traducteur dans le domaine de l'assurance. Chez nous, c'est plus varié. Nous avons aussi la chance d'être plus nombreux, ce qui nous permet de faire commencer quelqu'un au bas de l'échelle. Nous avons des traducteurs 1, 2 et 3 . Ensuite nous avons le réviseur. Nous faisons le recrutement de plusieurs façons. Par exemple, nous faisons paraître des annonces dans les journaux. M. Metz a expliqué ce que ça rapporte.

F. G. - Est-ce que l'annonce ne paraît que dans les journaux locaux ?

L. F. - Parfois. Si ces annonces ne rapportent pas, nous les faisons paraître dans la Presse par exemple ou le Droit; nous pouvons aussi annoncer dans les circulaires de l'A.T.I.O.

J. G. D. - Laquelle des formules vous semble la meilleure?

L. F. - Les journaux locaux.

F. G. - Qui se présente ?

L. F. - Nous avons des gens qui ont suivi des cours à l'université, « they majored in French ». Des candidats comme ça nous en avons huit sur dix.

D. L. - Il y a beaucoup de professeurs de français aussi.

L. F. - Toutes sortes de personnes se présentent qui ont fait de la traduction, soit à l'école, soit à la maison. Notre service du personnel fait une présélection et ne nous envoie que les gens qu'il croit être qualifiés. Nous rencontrons ces gens-là.

F. G. - Vous leur faites aussi subir un examen ?

L. F. - Si au cours de l'entrevue on voit que le candidat ne fait pas l'affaire, il ne subit pas l'examen. S'il fait l'affaire, il a à subir un examen écrit. Un candidat m'a déjà dit : "Moi j'aime ça la translation, mais j'ai un peu de difficulté à donner la bonne signifiance à mes sentences. » Avec un cas comme celui-là, on ne va pas plus loin. Une autre méthode de recrutement rapporte bien chez nous : j'ai demandé au chef du personnel de m'envoyer tous ceux qui, d'après lui, ont les qualifications nécessaires pour devenir traducteurs, que nous ayons un poste vacant ou non. Nous constituons ainsi un réservoir où nous puisons au besoin.

J. G. D. - Jusqu'à quel point le service du personnel est-il en mesure de vous aider? Chez nous le service du personnel nous dit parfois : «She is very good in English, I think she would make a very good' translator. » 
L. F. - J'ai expliqué au service du personnel nos exigences. Le « High School French» ou le «University French» quand la langue maternelle n'est pas le français, ça ne sert à rien. En cas de doute, ils me téléphonent. Lorsque des candidats intéressants se présentent et que nous n'avons pas de poste vacant dans notre service, je demande au bureau d'emploi d'essayer de trouver pour cette personne un poste dans la compagnie.

F. G. - Mademoiselle Lord, vous avez eu une expérience de recrutement récemment. Pouvez-vous nous en parler?

D. L. - J'ai d'abord fait paraître une annonce par l'entremise de l'A.T.I.O. Les gens qui ont répondu étaient tous trop qualifiés. J'ai ensuite fait paraître des annonces à Montréal et à Québec. Je me suis dit que j'avais probablement plus de chances en essayant directement dans le milieu francophone. Les résultats à Montréal n'ont pas été très bons. De Québec, j’ai reçu une foule de réponses.

L. F. - Les gens de Montréal hésitent à se déplacer, à venir s'établir de façon définitive à Toronto. Ils sont prêts à faire un stage pour perfectionner leur anglais, mais on peut difficilement les garder plus de six mois ou un an.

J. G. D. - Chaque fois que je suis allé chercher des traducteurs au Québec, je ne les ai jamais gardés longtemps. Je cherche maintenant à prendre des personnes qui sont ici depuis un an ou deux au moins.

F. G. - Est-ce cncorc vrai ce qu'on disait au sujet d'un traducteur du Québec qui venait s'établir à Toronto, à savoir que, baigné dans un milieu anglophone, il perdait, après une année ou deux de vie ici, ses qualités de rédacteur ?

J. G. D. - Je suis convaincu que non. Personnellement, je me suis amélioré en français. Il faut faire un effort constant pour éviter les anglicismes. Quand j'étais au Québec, j'employais beaucoup plus facilement des anglicismes qu'aujourd'hui et ce, sans le savoir. La plupart des francophones, surtout les traducteurs, vivent quand même en français, travaillent en français et parlent français à la maison. Vous avez maintenant la radio, la télévision françaises, et les cinémas. Toronto.

D. L. - Depuis les cinq dernières années, le français a fait son chemin à

F. G. - D'après vos propos, il semble que vous fassiez surtout de la traduction vers le français. Avez-vous l'occasion de traduire aussi vers l'anglais?

C. M. - Très peu. À peine cinq pour cent du volume total du travail.

F. G. - Est-ce qu'il s'agit de textes envoyés de l'extérieur que vous devez traduire vers l'anglais ou s'agit-il aussi de textes rédigés en français par vos clients ?

C. M. - Il y a les deux. Premièrement, il y a des lettres, mais il y en a relativement peu, parce que ça coûte assez cher de faire traduire des petites lettres de rien du tout par un service de l'extérieur. S'il y a un certain volume de correspondance, le client préfère embaucher une dactylo bilingue. Deuxièmement, il y a aussi des documents ou des textes scientifiques, mais c'est très peu volumineux.

D. L. - Nous avons beaucoup de lettres, en particulier des lettres du gouvernement du Québec qui rédige toute sa correspondance en français. Cependant je 
n'ai jamais à traduire les réponses vers le français ; je crois que l'entreprise répond en anglais.

J. G. D. - Il peut y avoir aussi des procès-verbaux de réunions et autres choses du même genre.

D. L. - Il y a aussi des contrats de vente.

L. F. - Le volume de traduction vers l'anglais chez nous a aussi augmenté considérablement depuis la Loi 22. La direction de Montréal, qui avait l'habitude de communiquer en anglais avec la direction de Toronto, communique maintenant en français. Nous traduisons donc les lettres provenant de Montréal vers l'anglais et les réponses en provenance de Toronto vers le français.

F. G. - Est-ce que vous traduisez vous-même d'autres langues que l'anglais et le français?

D. L. - On nous demande de le faire. À moi ils apportent toutes les langues; parce que je suis traductrice, je devrais pouvoir toutes les traduire. De l'espagnol, de l'allemand, du portugais.

F. G. - Disposez-vous d'un réservoir de traducteurs-pigistes ?

D. L. - Je consulte simplement le répertoire de l'A.T.I.O. ou encore je fais comme j'ai fait cette année: j'ai appelé une société de traduction pour lui demander si elle disposait de traducteurs dans telle et telle langue ; on m'a fourni des noms. J'ai fait demander des estimations. C'était pour le service des ventes, ils ont trouvé que c'était trop cher.

J. G. D. - Peut-être que dans votre domaine, Charles, vous allez avoir à traduire un mode d'emploi en japonais par exemple.

C. M. - Japonais, ça je ne touche pas, mais quand c'est quelque chose de très simple, je fais faire de l'allemand vers l'anglais. Si c'est technique, je n'y touche pas. Une de nos employés sait très bien l'espagnol. Un moment, on avait quelqu'un qui savait très bien l'italien; c'est à peu près les trois langues qu'on a le plus souvent. Si ça sort de là, alors on le donne à un spécialiste. Mais c'est très difficile pour nous de recommander un traducteur. Je le connais peut-être, mais pas la qualité de son travail.

J.G. D. - J'ai un client à la pige pour lequel je fais régulièrement de la traduction de l'anglais au français. Un jour il m'est arrivé avec un texte allemand à traduire vers l'anglais. Je lui envoie donc un traducteur que je connais. Par la suite mon client me téléphone pour me dire : «Méfie-toi, parce qu'il nous a fait une offre pour avoir nos traductions de l'anglais au français. »Il coupe les prix, que voulez-vous ? Mon client a été assez honnête pour me le dire; c'est embêtant parfois de recommander quelqu'un.

L. F. - J'aimerais maintenant proposer quelque chose en ce qui concerne le recrutement. J'établis des contacts avec les bureaux de traduction et certains services de traduction de Toronto. Si j'ai besoin de traducteurs, je communique avec ces gens. Ils font de même avec moi. Si un bon candidat se présente chez moi 
et que je n'aie pas de poste vacant, je le recommande ailleurs. S'il demande un salaire que je ne peux offrir, mais que sa compétence justifie pleinement, peut-être qu'ailleurs il pourrait être embauché. Il me semble donc que nous aurions peut-être avantage à établir et à maintenir de tels contacts ici à Toronto.

D. L. - Peut-être par l'A.T.I.O.

L.F. - Les demandes que j'ai faites par l'A.T.I.O. n'ont jamais rapporté ; la majorité des membres sont d'Ottawa et ne sont pas intéressés à venir travailler ici. Nous avons des traducteurs qui nous ont laissés pour un meilleur avenir, à Ottawa.

F. G. - Est-ce que le fait que chez vous le service et les bureaux soient relativement petits et offrent en somme peu de possibilités d'avancement n'entraîne pas des difficultés au moment du recrutement?

L. F. - Chez nous, j'ai demandé au service du personnel de faire une étude du marché une fois l'an pour voir quels salaires sont versés ailleurs. Si nos salaires sont trop bas par rapport à la moyenne, ils sont montés. Nous payons maintenant le salaire du marché.

F. G. - Vous ne pouvez quand même pas promettre à un traducteur un avancement rapide en quelques années. Vous avez du personnel en place qui risque d'être là pour longtemps.

L. F. - Un nouveau peut quand même, après un certain temps, devenir meilleur qu'un traducteur qui est en place depuis longtemps. Nous payons au rendement et d'après la qualité du travail et non d'après l'ancienneté.

F. G. - Parlons maintenant de la terminologie.

L. F. - Chez nous la terminologie est faite par l'équipe de traducteurs. Le travail est coordonné par le chef de service. Le réviseur et le traducteur travaillent ensemble. Si les opinions divergent sur un même terme, ils en discutent; s'ils ne peuvent s'entendre, ils viennent me voir: après avoir fait des recherches, nous tranchons. Le terme est adopté, on l'insère dans notre lexique.

F. G. - Vous procédez par lexiques. Avez-vous seulement un lexique général ou aussi des lexiques spécialisés?

L. F. — Nous avons les deux. Nos lexiques existent par souci d'uniformité et du mot juste.

F. G. - Est-ce que les traducteurs sont tenus de faire des fiches ou de noter le fruit de leurs recherches en cours de travail?

L.F. - Pas de la façon dont nous procédons. Le terme cherché par le traducteur est incorporé dans son lexique qui est sa propriété personnelle.

F. G. - Son lexique à lui est-il aussi le lexique de tout le monde ?

L. F. - Absolument.

J. G. D. - Si le traducteur s'en va, est-ce qu'il emporte son lexique ?

L. F. - Les traducteurs ne veulent pas se départir de cet outil. Il représente vingt-cinq ou trente années de recherches. 
J. G. D. - Chez nous il y a beaucoup de travail répétitif : nous avons donc fait des modèles qui servent de normes. Pour le reste, il y a déjà beaucoup de travail qui a été fait dans le domaine de l'assurance par la Régie de la langue française: c'est ce dont nous nous servons. Comme nous touchons à plusieurs autres domaines, il nous faut des dictionnaires spécialisés, c'est l'affaire de chacun. Nous nous sommes aussi procuré les travaux du Secrétariat d'État. Les traducteurs en ont fait venir pour eux-mêmes ; mais c'est dommage qu'ils ne soient plus gratuits, personne n'en fait plus venir. Peut-être que l'A.T.I.O. pourrait faire des pressions comme elle l'a déjà fait auparavant. Elle pourrait peut-être même se charger de la distribution. Ce serait au moins un service que l'A.T.I.O. rendrait.

F. G. - Les traducteurs gardent-ils pour eux-mêmes le fruit de leurs recherches?

J. G. D. - Chacun a son petit fichier personnel.

D. L. - Je me sers aussi beaucoup des cahiers de l'Office de la langue française et je fais moi-même des fiches. Lorsque le travail est assez spécialisé et qu'il demande beaucoup de recherches, je fais des fiches pour chacune des expressions difficiles.

C. M. - La documentation c'est un peu mon dada depuis toujours. Quand je suis entré chez Holt, nous avions à peu près une cinquantaine de livres, maintenant nouls en avons peut-être cinq cents. En principe, on a des dictionnaires sur à peu près tous les domaines. Il s'agit là des instruments de base. Nous recevons aussi toutes les revues techniques qui se publient au Québec. Il fut un temps où l'on gardait tout ça dans des classeurs, soigneusement. Quand on avait besoin de certains mots ou de certains renseignements, il fallait faire tout le dossier. Maintenant on a trouvé un système très pratique. On dépouille les revues : on découpe les articles et on les classe par sujets. Il y a aussi des lexiques dans ces revues techniques : aussitôt qu'on en trouve, on enlève les pages en question et on en tire des photocopies. On garde deux exemplaires de chaque lexique au cas où l'original se perdrait ou serait mal classé. À part cela, je prends relativement peu de notes sur mon travail. Il fut un temps où j'avais aussi un petit carnet noir. Mon collègue en a un. On n'y note pas tellement de renseignements techniques; c'est plutôt des choses qui échappent à la mémoire. La documentation demande beaucoup de travail et c'est justement là où nos trois dactylos participent. On les forme pour qu'elles puissent nous aider dans ce genre de travail.

F. G. - En réalité ce ne sont pas les traducteurs qui font le dépouillement, ce sont les dactylos.

C. M. - J'essaie quand même de leur montrer comment faire le travail, afin de les mettre sur la voie. Il faut savoir quoi chercher, savoir ce que nous avons déjà comme catégories, comme dossiers, etc. Très souvent vous mettez des choses de côté comme ça et pendant des années vous n'en avez pas besoin; mais tout à coup vous avez un travail pressé, sur les détergents par exemple, c'est là un vocabulaire très spécial qu'on ne trouve pas dans le Harrap's et si vous ne connaissez pas les termes, où les trouver à Toronto ? 
J. G. D. - C'est justement notre problème. Il peut nous arriver par exemple une demande de règlement, un cas d'invalidité. Le type peut avoir 30 ans, il a droit à des prestations d'assurance jusqu'à l'âge de 65 ans et à une rente d'invalidité. On nous fait une description du travail de l'invalide; pour savoir si c'est accidentel, s'il y a eu négligence de la part de l'employeur, on entre dans des descriptions techniques, des noms de machines de toutes sortes. Où se tourner pour obtenir de la documentation ? On n'a absolument aucun moyen, on fait ce qu'on peut. Dans le cas des nouvelles lois fiscales, on peut s'adresser tout simplement à l'Imprimeur de la Reine. Mais ici à Toronto, je ne sais si vous avez eu la même expérience que moi, il n'y a rien en français. Il faut tout faire venir d'Ottawa et, quand ça nous arrive, le travail est déjà remis, il est trop tard.

F. G. - Croyez-vous qu'une banque de terminologie que vous alimenteriez et qui vous fournirait des équivalents vous serait utile?

J. G. D. - Tout dépend du fonctionnement, du coût, de la disponibilité. Je ne crois pas que le terminal soit quelque chose qui prenne énormément d'espace. Et sous quelle forme viendrait la documentation en question?

F. G. - Vous pourriez recevoir sur imprimante ou sur écran cathodique l'information voulue.

L. F. - Il s'agirait d'une photo de fiche qui apparaîtrait sur un écran avec la définition et le terme?

F. G. - La définition, le contexte, la source, le domaine.

J. G. D. - Le terminal coûte un certain montant, disons mille dollars ; il doit aussi y avoir un certain prix d'abonnement pour la participation à la banque, de même qu'un tarif pour chaque interrogation. $\mathrm{Si}$ vous devez y avoir recours assez souvent, surtout dans un assez gros service, il faudrait faire le calcul pour savoir combien ça peut coûter.

C. M. - Il me semble avoir vu quelque part quatre mille dollars.

F. G. - Peut-être qu'à l'époque c'était exhorbitant; aujourd'hui, on dit que les prix baissent.

L. F. - Le système de transmission va en s'améliorant constamment.

F. G. - Il y a, en effet, accélération de la vitesse de réponse. Il est possible maintenant d'obtenir des transmissions téléphoniques très rapidement.

J. G. D. - Dans le cas d'une description de tâche par exemple, on n'a pas besoin de glossaire, on consulte directement la banque. Mais si dans certains domaines, il y avait moyen d'avoir les données sous forme de glossaires, ça serait déjà une bonne source d'économie pour le client.

F. G. - Est-ce que vous seriez prêts, de votre côté, à alimenter une banque en consignant sur des fiches le fruit de vos recherches quotidiennes et en fournissant vos données avec références à la banque ?

L. F. - Et qui jugerait et accepterait le terme? 
F. G. - Il pourrait y avoir quelque part un comité d'étude. Vous auriez sans doute aussi la possibilité d'uniformiser pour votre propre service. Vous pourriez avoir un moyen de savoir que pour une vedette donnée c'est tel équivalent plutôt que tel autre que votre service utilise.

J. G. D. - J'ai beaucoup de documentation sur l'assurance et quand on prend les glossaires de l'Office de la langue française, par exemple, c'est toujours les expressions les plus courantes qu'on y trouve. On dit toujours qu'ils évitent les mots difficiles ou compromettants. Les mots techniques, on ne les trouve nulle part.

L. F. - Il y a des mots qui peuvent avoir différentes définitions. L'équivalent que nous cherchons a une définition rare et non pas courante. Est-ce que la banque sera en mesure de nous fournir le terme qui recouvre cette définition?

F. G. - Si le terme est propre à votre service, de même que sa définition, c'est d'abord à vous de le mettre en banque. Si une autre entreprise du même genre que la vôtre a déjà fourni des données concernant le terme-problème, vous pouvez y avoir accès. De la même manière, d'autres sociétés pourront aussi avoir accès aux termes que vous aurez emmagasinés.

L. F. - Il peut donc y avoir deux ou trois définitions de différentes sources pour la même expression?

F. G. - C'est plus compliqué que cela, mais une banque de terminologie doit rassembler les différentes définitions d'un terme et les sources de chacune d'elles.

J. G. D. - Il faudrait envisager que s'il doit se faire un échange, ce ne soit pas à sens unique. Est-ce qu'une banque serait prête à donner certains privilèges aux gens qui collaboreront régulièrement? Il me semble que ce serait quelque chose qui stimulerait les traducteurs à participer. Dans le travail quotidien, on fait une recherche et, lorsqu'on a trouvé, on s'empresse de faire une fiche et de la mettre de côté ; mais pour commencer à communiquer ses informations, il faut aussi donner des sources, cela demande du temps : il faudrait donc un stimulant quelconque.

F. G. - Vous êtes tous spécialistes dans différents domaines. Seriez-vous prêts à participer à des comités nationaux ou internationaux de normalisation portant sur le vocabulaire de la discipline qui vous intéresse?

J. G. D. - Il y a toujours une question de coût. Dans une entreprise comme la nôtre, si on sait qu'il doit y avoir deux réunions, une à Québec et l'autre à Lyon, il faut prévoir cela dans le budget. Parce que c'est beaucoup plus difficile de faire des voyages lorsque le budget a été accepté. Je pense cependant que c'est assez facile de convaincre l'employeur qu'on a tout avantage à collaborer.

L.F. - Certains croient qu'un service devrait avoir un terminologue qui consacrerait tout son temps à la terminologie, à la préparation des fiches, des lexiques, des glossaires: je me demande si c'est possible dans l'entreprise privée. Nous avons beaucoup trop de travail à faire. Nous ne pourrions pas nous payer le luxe d'avoir une personne qui ne ferait que cela. Est-ce que ce serait un meilleur système que celui qui consiste à faire de la terminologie en groupe comme nous 
faisons chez nous? Avoir une personne qui ne fait que de la terminologie quand nous avons quinze pages à sortir dans trois jours, je regrette, mais il faudra que le terminologue se retrousse les manches, prenne sa machine et sorte le travail lui aussi.

F. G. - Vous voyez le terminologue comme étant aussi traducteur ?

L. F. - Ça dépend du volume de travail à faire. S’il n'y a pas de travail à faire, on peut très bien laisser le terminologue à sa recherche.

J. G. D. - Et encore il peut vous trouver les termes justes ou plus ou moins justes : entre deux bons termes, il faut choisir.

L. F. - II faut que le travail soit coordonné.

F. G. - C'est avec les traducteurs et le chef du service de traduction (qui coordonne le travail) que le terminologue tente d'uniformiser le vocabulaire.

L. F. - Le chef du service, à la lumière des recherches, tranche la question.

J. G. D. - Est-ce qu'il y a encore, surtout chez vous, Charles, des personnes qui vous disent : "Faites-vous du Parisian French ou du Quebec French?»

C. M. - La traduction, il faut le dire, c'est un domaine très mal connu. L'homme d'affaires moyen, ici à Toronto, ne sait pas que la traduction existe. Les seules personnes qui savent un peu ce que c'est que la traduction, ce sont les imprimeurs qui impriment du français et de l'anglais, à longueur de journée, puis des personnes dans de grandes entreprises qui ont des relations avec Québec, ou qui sont responsables de la publication de revues, de dépliants ou autres choses du genre. La secrétaire moyenne, dans une entreprise à Toronto, ne sait pas que la traduction existe.

L. F. - C'est vrai cela. Mais il y en a aussi qui pensent qu'il y a deux genres de traduction : "Canadian French» et «Parisian French». Récemment un de nos traducteurs nous a quittés pour occuper un poste dans une compagnie assez importante de Toronto, en informatique. D'autres personnes qualifiées se sont aussi présentées. Dès le moment où les personnes qui faisaient passer l'entrevue se sont rendu compte que certains candidats venaient de France, elles leur ont fermé les portes en disant: "We don't want Parisian French. We deal with the Canadian market, you must be French Canadian. »À mon sens, les écoles, les universités, ici à Toronto, sont la cause de cette attitude. On y dit aux élèves : «This is Parisian French.»Ce sont des professeurs anglophones qui enseignent le français aux élèves et ils disent enseigner le «Parisian French. » Les étudiants sortent de l'université avec l'idée qu'il y a deux français.

C. M. - Parfois on nous dit aussi : "Vos traductions sont très bonnes, mais elles sont trop bonnes. Le public ne comprend pas. » Par exemple, au point de vue terminologie technique, prenons le mot sand en anglais, nous le traduisons toujours par sabler: c'est le terme que tout le monde emploie au lieu de poncer. Nous savons très bien que dans la plupart des lexiques on trouvera poncer.

J. G. D. - Par contre, si vous écoutez tous les soirs à la radio l'émission Monsieur bricole, il vous parle toujours de poncer. Que choisir? Vous en trouverez toujours qui diront: "Nous on veut du bon français, pas du français de la rue. » 
Comme traducteurs nous cherchons toujours à nous protéger pour que, si on mettait nos traductions en doute, nous puissions nous justifier. D'autres par contre disent : «C'est du français de dictionnaire, on ne dit pas ça comme ça. » Il faut se brancher!

F. G. - Qui vous dit cela ?

J. G. D. - Tout le monde. Il peut s'agir d'un autre traducteur qui essaie de vous faire mettre les pieds dans les plats ou qui prêche pour sa paroisse. Il y a des maisons de traduction qui annoncent par exemple: "French tailored to Quebec market. »

C. M. - Ça ce n'est pas un défaut. Il faut tout de même que le traducteur d'ici s'adapte au marché québécois. Il y a certaines traductions pour lesquelles vous mettrez une expression pour la France que vous ne mettrez pas pour le Québec, et vice-versa.

J. G. D. - Même si une maison de traduction dit travailler plus pour le Québec que pour la France, je pense que dans tous les bureaux il y a les Européens et les Canadiens. Je pense qu'au point de vue éthique professionnelle, ce genre de concurrence serait à éviter. Le «Moi je suis plus français qu'un autre» ou le «Moi je suis plus québécois » entretient de vieux préjugés qui existent chez les clients, et peut-être plus ici qu'ailleurs.

D. L. - Quant à moi, je n'ai pas eu de plaintes ni de commentaires de ce côté-là. Je vais faire une différence par exemple quand je traduis le journal des employés. Le niveau de langue sera alors certainement moins élevé que pour une brochure ou quelque chose de plus sérieux.

L. F. - Certains nous ont dit que notre catalogue était trop français et qu'ils ne comprenaient pas. Pour éviter cela, je dis toujours à mes gens : «Si vous avez à choisir entre deux expressions, choisissez toujours la plus simple, mais ne choisissez pas une expression qui serait fautive. »

C. M. - Très souvent, ce qui se passe dans ces cas-là c'est que, pendant longtemps, il y a plusieurs termes qui coexistent. Tout à coup, il y en a un qui semble être adopté et qui prend le dessus.

L. F. - Lorsqu'un mot nouveau s'implante, il n'y a pas de problème. Mais quand on pense à un terme très courant comme fournaise à l'huile, c'est un terme que tout le monde emploie. Qu'est-ce qu'il faut choisir? Si vous essayez de vendre une chaudière au mazout allez-vous réussir? Le but du catalogue, c'est de vendre au client un article qu'il connaît, qu'il peut acheter et dont il a besoin. Nous employons donc fournaise à l'huile.

F. G. - Est-ce que vous avez accès à la documentation fournie par le client ? Est-ce qu'il vous envoie des explications autres que le texte à traduire?

L. F. - Nous, on obtient ce qu'on demande. Notre contact, c'est l'acheteur; parfois on lui demande de quoi il veut parler. Un autre contact peut être le chef du rayon. Ces gens collaborent sans difficulté. S'ils ne peuvent rien fournir, nous allons directement voir le fabricant. Nous obtenons d'ailleurs de celui-ci beaucoup de documentation et très souvent en français. 
C. M. - Un nouveau client qui vient chez nous veut nous mettre à l'épreuve. Il veut voir par exemple si notre traduction va être suffisamment bonne pour être acceptée par le directeur des ventes de Montréal ou par les vendeurs ; il veut aussi savoir si elle sera meilleure, moins chère et faite plus rapidement que celle qu'il avait avant. À ce moment-là, il ne nous donne pas de documentation. Mais s'il nous en faut, nous la demandons. Nous l'obtenons, mais quelquefois il faut tirer l'oreille du client.

F. G. - À la Noranda, est-ce que c'est difficile d'obtenir de la documentation ?

D. L. - Il n'y a pas vraiment de gros problèmes de ce côté. La piupart du temps, le client n'envoie pas volontairement la documentation. À ce moment-là, je la demande. S'il y en a, on me l'envoie. Lorsqu'une traduction a déjà été faite, je dois la demander; on ne me l'enverra pas. Il y a une éducation qui se fait. Quand on m'envoie des traductions sans documentation et que j'en demande une première fois, par la suite, on n'hésite pas à en envoyer.

L. F. - Ils ne savent pas qu'il faut envoyer de la documentation. La plupart du temps, ils pensent que la traduction se fait sans références.

J. G. D. - Il arrive aussi qu'à l'intérieur de la compagnie, les rédacteurs oublient que leur texte doit sortir en français. L'année dernière, la secrétaire de la compagnie m'a téléphoné la veille de l'assemblée annuelle pour me dire qu'il fallait traduire le rapport annuel. Heureusement que j'y avais pensé et que c'était chose faite et même imprimée. Si je n'étais pas allé au-devant des coups, le rapport annuel, je l'aurais donc reçu la veille de l'assemblée annuelle. Toronto.

F. G. - Si on abordait le sujet délicat des relations entre traducteurs à

J. G. D. - Du manque de relations entre traducteurs. C'est presque nul. Il fut un temps où les relations entre les membres de l'A.T.I.O. marchaient assez bien. C'est d'ailleurs par l'entremise de 1'A.T.I.O. que j'ai rencontré tous les traducteurs de Toronto. J'en connais plusieurs assez bien pour les appeler par leur prénom et ne pas hésiter à leur téléphoner pour leur demander des renseignements ou entretenir certains liens d'amitié. Tout cela semble être en train de disparaître maintenant. Les nouveaux membres se demandent ce que l'A.T.I.O. donne à part son petit bulletin : Informatio. C'est l'inactivité totale, il n'y a aucune rencontre, il n'y a aucun lien.

D. L. - Nous avions une réunion une fois par année pour rencontrer les nouveaux membres juste avant la réunion annuelle.

L. F. - Nous nous rencontrions une fois par année et c'était tout.

J. G. D. - À un point tel que je me suis demandé... Je ne voudrais pas créer une situation qui a existé au Québec, soit deux ou trois groupes, mais il semble que ce serait presque le moyen de nous réunir. Si les réunions de l'A.T.I.O. doivent s'autofinancer à Toronto, je l'ai vécu, ça ne peut pas marcher. Très souvent, j'ai dû avancer les frais de la réunion de ma poche pour ne me faire rembourser qu'un mois après par l'A.T.I.O. Nous devions donc espacer les réunions, parce qu'on nous disait : « À Ottawa ça s'autofinance, il faut que ça s'autofinance à Toronto.» 
Lorsqu'il s'agit de louer une salle, que vous ayez quinze ou soixante-quinze membres, c'est le même prix. Si le traducteur doit toujours débourser cinq, six, ou sept dollars pour aller à une réunion de l'A.T.I.O... Il y en a beaucoup d'ailleurs qui sont mal payés, les débutants surtout. Plusieurs pourraient se le permettre, mais il y en a beaucoup qui sont chiches. Il y a de la paresse aussi.

F. G. - Le peu d'échanges qui se fait ici entre les traducteurs se situe-t-il au niveau personnel ou professionnel?

C. M. - C'est quand même plutôt pour des questions professionnelles, par exemple quand vous avez besoin de renseignements sur quelqu'un que vous voulez embaucher.

F. G. - Vous êtes tous membres de l'A.T.I.O.?

J.G.D. - Tous membres, mais tous des membres ignorants de ce qui se passe dans l'A.T.I.O.

F. G. - Vous trouvez donc que le service fourni par l'A.T.I.O. est quand même assez mince.

J. G. D. - Il est nul pour les gens de Toronto.

L. F. - Malgré tout cela, nous avons quand même de nouvelles candidatures. Une personne chez moi a rempli sa demande hier pour l'examen. Tous veulent faire partie de l'A.T.I.O.

J. G. D. - Aussi longtemps qu'ils n'en font pas partie. Ensuite ils commencent à se poser des questions : «Qu'est-ce que ça me donne? Il y a trois ans que je paye des cotisations et $\mathrm{j}$ 'ai simplement reçu quelques feuilles de papier. $\gg \mathrm{Si}$ on demande à ces traducteurs qui est le président de l'A.T.I.O., ils ne le savent même pas.

L. F. - J'encourage très fortement mes traducteurs à s'inscrire. Je leur dis que ça ne leur rapportera peut-être pas grand-chose immédiatement, mais que ça les qualifie, les identifie comme traducteurs professionnels, les élève au-dessus du traducteur amateur, les met dans une place à part. Nous avons plusieurs membres de notre personnel qui sont membres de l'A.T.I.O.

J. G. D. - Chez nous nous payons, pour les personnes qui veulent devenir membres de l'A.T.I.O., leur droit d'examen et leur cotisation annuelle.

C. M. - Et au début, on augmentait le salaire d'un trađucteur aussitôt qu'il devenait membre.

J. G. D. - Ce qui est arrivé, c'est qu'à ce moment-là, il y a eu un réajustement des salaires pour tout le monde. C'est une coïncidence. Il faut dire cependant que mes activités dans l'A.T.I.O. ont beaucoup aidé. J'ai présenté à mes patrons l'échelle des salaires des traducteurs d'Ottawa; de leur côté ils se sont aussi informés. Si j'avançais un chiffre, ils étaient en mesure de le vérifier et voyaient bien que ce n'était pas des histoires que je leur racontais. Au traducteur qui venait me demander: "Qu'est-ce que ça me donne d'être membre de 1'A.T.I.O. ?». Je répondais : "L'A.T.I.O, ne vous l'a peut-être pas donné directement, mais est-ce 
que vous avez remarqué jusqu'à quel point votre salaire a augmenté depuis quelques années. » Je me suis énormément intéressé à l'A.T.I.O., j'ai invité plusieurs personnes à en faire partie ; à l'époque je pouvais fournir des renseignements, maintenant je ne suis plus en mesure de le faire. Si au moins on avait un compte rendu des réunions du Conseil. $M$. Gérard Gauthier soulève dans la lettre qu'il nous a envoyée un point qui est à la mode actuellement : l'aménagement paysager. Au cours des années, c'est allé en se détériorant. Il fut un temps où la traduction se faisait dans des locaux fermés; ensuite il y a eu les cloisons; puis on a fait disparaitre les cloisons pour les remplacer par des écrans. Si on veut parler à un employé de questions confidentielles, il faut toujours le faire à voix basse. Si quelqu'un vient, tout le monde est dérangé. En principe, vous n'êtes pas supposé entendre ce qui se passe à plus de six pieds, mais en réalité, vous entendez ce qui se passe à trente ou quarante pieds.

L. F. - Chez Simpsons, ils ont un immeuble assez neuf et leurs bureaux sont paysagers; les traducteurs se font face, le directeur lui-même fait face à un autre. Chez nous, j'ai exigé quatre murs et une porte afin que le traducteur se concentre uniquement sur son travail et ne soit pas dérangé par le va-et-vient. Eaton est en train de construire ici un très grand complexe. Ils avaient l'intention d'installer des bureaux à cloisons mobiles; j'ai fait valoir que des cloisons qui n'isolent pas le traducteur, ce n'est pas pratique, et que non seulement le va-et-vient, mais aussi les discussions des autres, les téléphones et le simple fait de faire face à une autre personne en travaillant empêchent de se concentrer. J'ai demandé que chaque traducteur ait son bureau particulier et c'est ce qu'ils nous font présentement.

J. G. D. - C'est bien qu'ils aient accepté ce que vous disiez. Quand ils ont fait le changement chez nous, j'ai cherché à obtenir que les traducteurs soient séparés, mais ça n'a pas marché.

L. F. - Tous nos traducteurs préfèrent des bureaux. J'ai demandé leur opinion : ils sont unanimes.

D. L. - Je viens tout juste d'avoir un bureau. J'en ai demandé un pendant trois ans et si j'en ai un, c'est parce que d'autres employés ont déménagé. Je suis la seule femme à avoir un bureau : c'est une grande faveur qu'on m'a faite. Mon adjoint occupe mon ancien emplacement. Maintenant c'est moi qui aurai des problèmes, parce que lui aussi va vouloir son bureau.

F. G. - De l'échange que nous avons eu, je voudrais savoir ce que vous avez retiré et $\mathrm{j}$ 'aimerais que vous formuliez des voux, si vous en sentez le besoin.

J. G. D. - Il y a d'abord un manque de communication entre les traducteurs et leur association. C'est pàr elle qu'ils peuvent entretenir des relations entre eux et obtenir des renseignements. Il y a aussi la question de la documentation; c'est une question de distance : une banque de terminologie serait sans doute la solution. Il y a encore l'ignorance du client qui n'a pas tellement d'idée de ce que peut-être la traduction.

F. G. - Est-ce qu'une campagne d'information bien orchestrée, faite par une association comme l'A.T.I.O., pour expliquer dans les journaux par exemple ce que 
c'est que la traduction, ne pourrait pas sensibiliser les clients éventuels et les faire changer d'attitude?

L. F. - Deux groupes de gens sont représentés ici : les traducteurs de services de traduction d'entreprises et les traducteurs d'entreprises de traduction, comme M. Metz. Nous avons constaté que les problèmes d'un groupe sont aussi les problèmes de l'autre. Nous avons constaté qu'il y a une lacune à Toronto, un manque de contact entre les traducteurs, disons même un manque total de contact. L'A.T.I.O. peut être un moyen de rassembler les gens; mais ça ne devrait pas être le seul moyen. Il me semble qu'on devrait améliorer cette situation, peut-être en prenant nous-mêmes la chose en main et en communiquant un peu plus sur le plan professionnel. Moi je suis prêt à le faire. Peut-être qu'en établissant un contact professionnel cela débouchera sur une coopération plus étroite entre les traducteurs de Toronto.

D. L. - C'est assez difficile d'ajouter quelque chose à ce qui a déjà été dit. Je déplore aussi le manque de communication entre les traducteurs de l'Ontario. Il a été mentionné une espèce de banque de demandes d'emploi qu'on pourrait partager avec les autres services de traduction.

F. G. - Quels pourraient être les mécanismes de cette banque ?

D. L. - Je ne sais pas, mais ça pourrait encore être fait par l'entremise de l'A.T.I.O. qui pourrait coordonner.

J. G. D. - Je pense que ça nous prendrait un secrétariat à Toronto. On ne nous envoie même plus les procès-verbaux des réunions de l'A.T.I.O. Auparavant, si on voulait des renseignements, il nous suffisait de téléphoner à Charles Metz, il avait les procès-verbaux et pouvait nous donner les renseignements voulus, tandis que maintenant on a cessé de lui envoyer ces choses-là. Même s'il les recevait un mois en retard, on savait quand même ce qui se passait.

C. M. - Si je peux émettre un vou, c'est que la banque de terminologie se fasse bientôt et sous n'importe quelle forme. Qu'elle nous arrive de Québec, de Montréal ou d'Ottawa, cela nous est égal. Qu'elle existe, qu'elle soit accessible au point de vue financier, et nous sommes prêts à y participer.

F. G. - Sur ce vou rempli d'espoir, au nom de Meta, je vous remercie d'avoir participé avec tant d'enthousiasme à cette petite réunion de famille. 